\title{
DETERMINAÇÃO DA 2,5-HEXANODIONA EM AMOSTRAS DE URINA SUBMETIDAS À HIDRÓLISE ÁCIDA: IMPORTÂNCIA DA COLUNA CROMATOGRÁFICA E DO pH DO MEIO
}

\author{
Márcia Martins Barroca, Josianne Nicácio Silveira e Edna Maria Alvarez-Leite* \\ Faculdade de Farmácia, Universidade Federal de Minas Gerais, Av. Olegário Maciel 2360, 30180-112 Belo Horizonte - MG
}

Recebido em 1/2/02; aceito em 14/2/03

\begin{abstract}
2,5-HEXANODIONE DETERMINATION IN URINE SAMPLES SUBJECTED TO ACID HYDROLYSIS: RELEVANCE OF THE CHROMATOGRAPHIC COLUMN AND THE pH. Acidic hydrolysis of samples is frequent in urinary 2,5-hexanodione determination. This hydrolysis should be performed under proper conditions, in order to avoid interference, such as the presence of 2,5-dimethylfurane and 2-acetylfurane. The results of the present work, as well as data from the literature, show that the use of non-polar or slightly polar chromatographic columns of $30 \mathrm{~m}$ length is an essential condition for 2,5-HD determination in an acid hydrolysed urine sample. In the same way the $\mathrm{pH}$ should be kept between 0.3 and 0.5 . The mean concentrations of 2,5-HD in samples submitted to hydrolysis $(0.50 \pm 0.28 \mathrm{mg} / \mathrm{g}$ of creatinine) was about 3 times higher than those found in the samples without acid hydrolysis $(0.19 \pm 0.3 \mathrm{mg} / \mathrm{g}$ of creatinine $)$.
\end{abstract}

Keywords: urinary 2,5-hexanodione determination; acid hydrolysis; analytical conditions.

\section{INTRODUÇÃO}

A determinação cromatográfica da 2,5-hexanodiona (2,5-HD) urinária como biomarcador de exposição ao $n$-hexano é um exemplo claro de como as condições analíticas podem influenciar os resultados de uma monitorização biológica de indivíduos expostos ao solvente.

Muito tem-se discutido sobre a necessidade ou não de ser realizada a hidrólise ácida da urina, objetivando liberar a 2,5-HD excretada conjugada. Quando esta hidrólise é realizada, os níveis deste biomarcador podem ser de 3 a 6 vezes maiores do que os encontrados nas mesmas amostras analisadas sem hidrólise, não só pela liberação do composto da forma conjugada mas, também, pela transformação in vitro de outros metabólitos urinários do $n$-hexano, como por exemplo o 4,5-diidroxi-2-hexanona (4,5-DH-2-H) em 2,5-HD ${ }^{1-3}$. Embora este fato aumente de maneira importante a sensibilidade e a especificidade da análise, é essencial que as condições analíticas sejam bem definidas, evitando-se resultados super ou subestimados do biomarcador. Sabe-se que, em hidrólises ácidas realizadas em $\mathrm{pH}$ próximos a 2,0, a 4,5-diidroxi-2-hexanona se transforma em 2,5 dimetilfurano (2,5-DF), subestimando a concentração total da 2,5HD encontrada ${ }^{1,2}$.

Outro ponto importante a ser considerado é a formação do composto 2-acetilfurano (2-AF), quando amostras de urina são submetidas à hidrólise ácida. Este composto, embora não seja metabólito do $n$-hexano, possui um tempo de retenção muito próximo ao da 2,5HD e poderá ser quantificado como tal, se as condições cromatográficas não forem capazes de separá-los efetivamente ${ }^{4}$.

O presente trabalho buscou verificar se, nas hidrólises ácidas realizadas em uma faixa de $\mathrm{pH}$ entre 0,3 e 0,5 , haveria a formação do composto 2,5-dimetilfurano. Foi avaliada, também, a efetividade de colunas cromatográficas na separação da 2,5-HD e do interferente 2-AF.

A 2,5-HD foi analisada por cromatografia gasosa utilizando-se detector por ionização em chama (CG-DIC). Para verificar se as con-

*e-mail: alem@dedalus.lcc.ufmg.br centrações encontradas eram realmente de 2,5-HD, ou seja, para confirmar a ausência de interferentes tais como o 2-DF e 2-AF, as alíquotas de urina foram analisadas, também, por cromatografia gasosa com detector de massas (CG-EM).

\section{PARTE EXPERIMENTAL}

\section{Material e métodos}

Colunas cromatográficas testadas

- HP-1 (12 m x 0,2 mm x 0,33 $\mu \mathrm{m}$ - metil silicone),

- HP-50+ (15 m x 0,53 mm x $1 \mu \mathrm{m}-50 \%$ fenil, $50 \%$ metil siloxano),

- DB-210 (30 m x 0,25 mm x 0,25 $\mu \mathrm{m}$ - 50\% trifuoropropil-50\% metil-siloxano),

- $\operatorname{HP}-20 \mathrm{M}$ (25 m x 0,32 mm x 0,3 $\mu \mathrm{m}$ - polietilenoglicol $20 \mathrm{M}$ ).

\section{Soluções padrões}

- de 2,5-HD em diclorometano (0,10 a $50 \mathrm{mg} / \mathrm{L})$ e

- de 2,5-HD, 2-AF e metilisobutilcetona (MIC) em diclorometano (3,0 $\mathrm{mg} / \mathrm{L})$.

\section{Amostragem}

- " "pool” de urina de indivíduos não expostos ao $n$-hexano (n=12), - amostras de urina de indivíduos expostos durante $8 \mathrm{~h} /$ dia ao solvente, em condições variadas de concentração e frequiência. As amostras foram colhidas ao final da exposição.

\section{Análise das amostras de urina}

O método de Kawai et al..$^{4}$ com pequenas modificações ${ }^{5}$ foi utilizado no presente trabalho e está esquematizado a seguir: transferir $2 \mathrm{~mL}$ de urina para tubos de vidro com tampa rosqueável, adicionar $200 \mu \mathrm{L}$ de $\mathrm{HCl} 50 \%$ (pH do meio entre 0,3 a 0,5) e levar para banho de água fervente por $30 \mathrm{~min}$. Adicionar $2 \mathrm{~mL}$ de diclorometano contendo MIC na concentração de $3 \mathrm{mg} / \mathrm{L}$. Agitar por $3 \mathrm{~min}$ (2000 rpm) 
e centrifugar por $15 \mathrm{~min}$ (3000 rpm). Injetar $1 \mu \mathrm{L}$ no CG-DIC e volume igual no CG-EM.

Quando se objetivava analisar as amostras de urina sem hidrólise ácida, as etapas de adição de $\mathrm{HCl}$ e aquecimento eram suprimidas.

\section{Condições cromatográficas}

CG-DIC: CG da HP, modelo 5890 série II; detector por ionização em chama; coluna DB-210; temperaturas do injetor a $230{ }^{\circ} \mathrm{C}$ e do detector a $235^{\circ} \mathrm{C}$; temperatura da coluna, programada, sendo a inicial de $60^{\circ} \mathrm{C} / 2 \mathrm{~min}$, rampa de aquecimento de $10^{\circ} \mathrm{C} / \mathrm{min}$ até $127^{\circ} \mathrm{C}$ e $40{ }^{\circ} \mathrm{C} / \mathrm{min}$ até a temperatura final de $200{ }^{\circ} \mathrm{C}$, mantida por $2 \mathrm{~min}$. Tempo de equilíbrio do forno: 0,5 min; ganho: 2 ; injeção: modo "split" 1/18. Gás de arraste: hélio, vazão de $1 \mathrm{~mL} / \mathrm{min}$.

CG-EM: CG da Shimadzu ${ }^{\circledR}$; modelo GC-17A; detector de massas por impacto de elétrons-quadrupolo. Programação do detector: faixa de massas: 40 a 500; intervalo da varredura: $2 \mathrm{~s}$; velocidade da varredura: 1000; tempo do corte do solvente: $2,50 \mathrm{~min}$; tempo inicial de aquisição: 2,8 min; tempo de corrida: 12,52 min; ganho do detector: $1,50 \mathrm{kV}$; monitor: modo espectro. As demais condições cromatográficas foram as mesmas utilizadas para o CG-DIC.

\section{Determinação da creatinina urinária}

A determinação da creatinina urinária foi realizada em aparelho de automação, Cobas Mira Plus - Roche ${ }^{\circledR}$, utilizando-se o método cinético de Jaffé - Kit Doles ${ }^{\circledR}$.

\section{RESULTADOS E DISCUSSÃO}

\section{Influência do pH do meio}

As amostras de urina de indivíduos expostos, hidrolisadas em pH 0,3 e 0,5, foram analisadas por CG-DIC. Os valores de 2,5-HD não foram diferentes em função do $\mathrm{pH}$ em que foi realizada a hidrólise. A análise das amostras por CG-EM confirmou a ausência do composto 2,5-dimetilfurano, indicando a adequação desta faixa de $\mathrm{pH}$ para a realização do tratamento hidrolítico (Figura 1).

\section{Tipo de coluna cromatográfica}

Colunas apolares ou medianamente polares são as mais indicadas para a separação adequada da 2,5-hexanodiona e 2-acetilfurano ${ }^{4,6,7}$; uma coluna apolar (HP-1, $12 \mathrm{~m}$ ) e outra de média polaridade (HP-50+, $15 \mathrm{~m}$ ) foram testadas. Observou-se que a separação entre 2,5-HD e 2-AF não foi adequada em nenhuma das duas, pois os compostos apresentaram o mesmo tempo de retenção nas mais variadas condições de vazão do gás de arraste e temperatura do forno. Em contrapartida, o uso da coluna polar HP-20M, 25 m, também não conseguiu separar os compostos, confirmando os estudos de Kawai et al. ${ }^{4}$.

Resultados opostos foram encontrados, entretanto, com o uso de outra coluna polar (DB-210, $30 \mathrm{~m}$ ) que conseguiu a adequada separação dos compostos, demonstrada pelos tempos de retenção obtidos após análise em CG-DIC e confirmados por CG-EM (Figura 1). A polaridade da DB-210 (fase estacionária 50\% trifuoropropil-50\% metil-siloxano) é menor do que a da HP 20M (fase estacionária polietilienoglicol) e este fato, aliado ao maior comprimento da coluna DB-210, pode ser a explicação para o resultado diferente encontrado em relação à separação dos compostos.

A adequação da coluna DB-210 para a análise de 2,5-HD em amostras hidrolisadas não está totalmente de acordo com a literatura consultada. Saito et al. ${ }^{7}$ testaram várias colunas cromatográficas para a determinação da 2,5-HD em urina submetida à hidrólise ácida e não indicaram a DB-210 como sendo uma das adequadas, pois os autores observaram interferência de outros componentes urinários na análise cromatográfica. Esta divergência entre os resultados poderia ser explicada pela diferença nas características das duas colunas DB-210 utilizadas. Saito et al. ${ }^{7}$ empregaram uma "megabore" de $15 \mathrm{~m}$ de comprimento, características estas que podem determinar uma menor separação dos compostos quando comparada com a DB210 de 30 m utilizada aqui.

A coluna DB-210 de $30 \mathrm{~m}$, empregada no presente trabalho, mostrou-se mais eficiente para a separação dos dois compostos do que a coluna utilizada no método original de Kawai et $a l .^{4}$, ou seja a DB-1, $60 \mathrm{~m}$ x 0,25 mm x 0,25 $\mu \mathrm{m}$. Os tempos de retenção encontrados foram, na DB-210: 2,5-HD = 8,5 min e 2-AF = 6,4 min (Figura 1); na DB-1: $2,5-\mathrm{HD}=18,44 \mathrm{~min}$. e $2-\mathrm{AF}=18,23 \mathrm{~min}$.

Uma vez otimizadas as condições analíticas, as amostras de urina dos indivíduos expostos ao $n$-hexano $(\mathrm{n}=16)$ foram analisadas paralelamente pelo método com e sem hidrólise (Figura 2). Pode-se observar que, nas amostras analisadas pelo método com hidrólise, tanto a concentração de 2,5-HD $(0,59 \pm 0,28 \mathrm{mg} / \mathrm{g}$ de creatinina) quanto a mediana $(0,6 \mathrm{mg} / \mathrm{g}$ de creatinina) foram cerca de 3 vezes superiores aos valores obtidos quando as mesmas urinas foram analisadas pelo método sem hidrólise (média: $0,19 \pm 0,13 \mathrm{mg} / \mathrm{g}$ de creatinina, mediana: $0,16 \mathrm{mg} / \mathrm{g}$ ).

Alíquotas de todos os extratos obtidos de urinas analisadas com e sem hidrólise ácida foram injetadas no CG-EM e em nenhuma delas foi detectada a presença do composto 2,5-dimetilfurano e do interferente 2-acetilfurano.

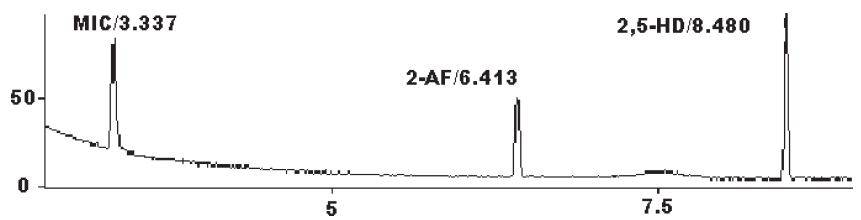

Figura 1. Cromatograma obtido após a injeção de solução padrão contendo 2,5-HD, 2-AF e MIC (todos na concentração de 3,0 mg/L) no aparelho $C G$ EM-17A QP 5000, detector de massas por impacto de elétrons-quadrupolo; programação do detector: faixa de massas: 40 a 500; intervalo da varredura: 2 s; velocidade da varredura: 1000; tempo do corte do solvente: $2,50 \mathrm{~min}$; tempo inicial de aquisição: 2,8 min; tempo de corrida: 12,52 min; ganho do detector: 1,50 kV; monitor: modo espectro
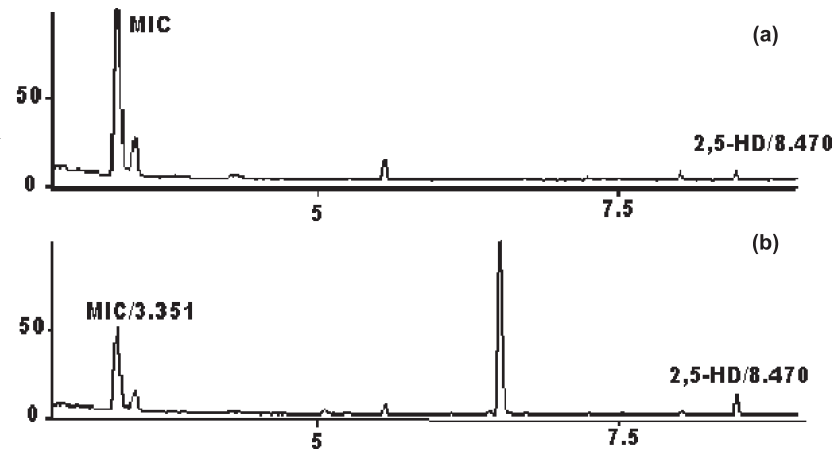

Figura 2. Cromatograma obtido após a análise de amostra urinária de indivíduo exposto ao n-hexano: (a) método sem hidrólise, (b) método com hidrólise ácida. Aparelho CG-EM-17A QP 5000, detector de massas por impacto de elétrons-quadrupolo, programação do detector: faixa de massas: 40 a 500; intervalo da varredura: 2 s; velocidade da varredura: 1000; tempo do corte do solvente: 2,50 min; tempo inicial de aquisição: 2,8 min; tempo de corrida: $12,52 \mathrm{~min}$; ganho do detector: $1,50 \mathrm{kV}$; monitor: modo espectro 


\section{AGRADECIMENTOS}

O autores agradecem à Fundação de Amparo à Pesquisa do Estado de Minas Gerais-FAPEMIG, pelo financiamento recebido

\section{REFERÊNCIAS}

1. Cardona, A.; Marhuenda, D.; Marti, J.; Brugnone, F.; Roel, J.; Perbelini, L.; Int. Arch. Occup. Environ. Health 1993, 65, 71.
2. Cardona, A.; Marhuenda, D.; Prieto, M. J.; Martí, J.; Periago, J. F.; Sánchez, J. M.; Int. Arch. Occup. Environ. Health 1996, 68, 88.

3. Van Engelen, J. G. M.; Kezic, S.; Haan, W.; Opdam, J. J. G.; Wolff, F. A.; J. Chromatogr, B 1995, 667, 233.

4. Kawai, T.; Yasugi, T.; Mizunumz, K.; Horiguchi, S.; Uchida, Y.; Iwami, O.; Iguchi, H.; Ikeda, M.; Int. Arch. Occup. Environ. Health 1991, 63, 213.

5. Barroca, M. M.; Silveira, J. N.; Alvarez Leite, E. M.; RBCF 2000, 63, 253.

6. Kawai, T.; Yasugi, T.; Mizunumz, K.; Horiguchi, S.; Uchida, Y.; Iwami, O.; Iguchi, H.; Ikeda, M.; Int. Arch. Occup. Environ. Health 1991, 63, 285.

7. Saito, I.; Shibata, E.; Huang, J.; Hisanaga, N.; Ono, Y.; Takeuchi, Y.; Br. J. Ind. Med. 1991, 48, 568 